\title{
A SELF-RECOVERING RAKE RECEIVER FOR ASYNCHRONOUS CDMA SYSTEMS
}

\section{Murat Torlak}

\author{
Department of Electrical Engineering \\ The University of Texas at Dallas \\ Richardson, TX 75083, USA \\ torlak@utdallas.edu
}

\author{
Brian L. Evans \\ Dept. of Electrical \& Computer Engineering \\ The University of Texas at Austin \\ Austin, TX 78712 USA \\ bevans@ece.utexas.edu
}

\begin{abstract}
In asynchronous CDMA systems, transmission over frequency-selective channels subjects the signal to interchip interference and intersymbol interference, both of which cause multiple access interference (MAI). MAI cannot be easily eliminated without the knowledge of the channel parameters. Both blind adaptive and subspace based multiuser receivers have been proposed to eliminate the interference. In this paper, we propose a self-recovering RAKE receiver that does not require training sequences or knowledge of interfering user signature waveforms. The contribution of this paper is the combination of constant modulus algorithm and previously derived constrains. The constrains depend on the multipath structure of the desired signal. The proposed receiver converges faster than the blind adaptive multiuser receivers. Its fast convergence and low complexity make it well suited for rapidly time-varying mobile radio frequency selective fading channels. By simulation, we compare the proposed method with blind adaptive constrained LMS RAKE receiver and minimum output energy receiver.
\end{abstract}

\section{INTRODUCTION}

Multiple access interference (MAI) and multipath fading which causes interchip interference (ICI) are the limiting factors of the performance of code division multiple access (CDMA) systems. Although a conventional RAKE receiver can coherently combine multipath, it is still limited by the MAI due to its inability to perform any interference mitigation.

Recent research has been devoted to the blind multiuser receivers and subspace-based signature waveform estimation schemes that exploit the fact that the structure of the MUI and ICI can be exploited to achieve better performance and, more importantly higher capacity $[1,2]$. The primary motivation of employing a blind method is to avoid the requirement of a training sequence. Due to the use of subspace decomposition, these methods require heavy computation. On the other hand, blind adaptive multiuser receivers have been proposed to reduce this computational load while showing performance close to that of the MMSE receiver [3]. The blind algorithm proposed in [4] is effective in MAI without necessarily exploiting multipath diversity. In order to effectively exploit multipath diversity, constrained adaptive algorithms have been derived to combine all the multipath components while minimizing MAI at the receiver's output [5]. These blind methods are potentially of great importance to practical applications in multiuser detection.

The contribution of the current paper is the combination of constant modulus algorithm and previously derived constrains. The constrains depend on the multipath structure of the desired signal. The proposed receiver converges faster than the constrained adaptive multiuser receivers [5]. Its fast convergence and low complexity make it well suited for rapidly timevarying mobile radio frequency selective fading channels. By simulation, we compare the proposed method with blind adaptive constrained LMS RAKE receiver and minimum output energy receiver.

\section{MODEL DESCRIPTION}

To simplify the description of the problem, we temporarily ignore the noise and leave the accommodation of noisy data for later discussion. We consider an asynchronous CDMA (A-CDMA) baseband signal with a single receiver from $\mathrm{P}$ users:

$$
x(t)=\sum_{i=1}^{P} \sum_{n=-\infty}^{\infty} s_{i}(n) g_{i}\left(t-n T_{s}\right)
$$

where subscript $i$ denotes the user index; $P$ is the number of users; $\{s(n)\}$ are the information symbols from 
a finite set of alphabets, e.g., $\{ \pm 1\} ; T_{s}$ is the symbol duration. Assume that $\left\{c(1), c(2), \cdots, c\left(L_{c}\right) ; c(k)= \pm 1\right\}$ is the prearranged spreading code of this user, $L_{c}$ is the code length. The signature waveform of $i^{t h}$ user with

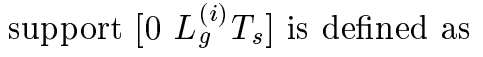

$$
g_{i}(t)=\sum_{k=1}^{L_{g}^{(i)} L_{c}} c_{i}\left(k-k_{i}\right) h_{i}(t-k T)
$$

where $T$ is the chip duration; $k_{i}$ is the chip delay index; $\left\{h_{i}(t)\right\}$ are the channels which represent the multipath fading environment between users and the receiver.

A multipath fading channel can be described as

$$
h(t)=\sum_{q=1}^{L_{d}} \alpha_{q} p\left(t-\tau_{q}\right)
$$

where $L_{d}$ is the total number of paths. $\tau_{q}$ and $\alpha_{q}$ are the associated delay and complex gain of the $i^{t h}$ path, respectively. $p(t)$ is the pulse function (e.g., raisedcosine pulse). We may assume that the channel order $L \ll L_{c}$ since the maximum delay spread of the channel is usually insignificant in relative to the symbol period $T_{s}$. Thus, depending upon the relative time delay and the channel length, the signature waveforms may last at most three symbol periods. However, for the sake of presentation simplicity, we will assume $L_{g}^{(i)}=2$ for each user throughout the paper.

To reveal the relations between input and output data structures, let us represent $x(t)$ in the discrete form. Sampling $y(t)$ at the chip rate and we obtain

$$
x(l)=\sum_{i=1}^{P} \sum_{n=-\infty}^{\infty} s_{i}(n) g_{i}\left(l-n L_{c}\right)
$$

The discrete version of composite spreading waveform $g(t)$ is given by

$$
g_{i}(l)=\sum_{k=1}^{L} h_{i}(k) c_{i}\left(l-k+k_{i}\right), \quad l=1, \cdots, 2 L_{c} .
$$

where $k_{i}$ is the chip delay index which is known to the receiver. Using the $i$ th user code matrix defined in [1] as

$$
\mathbf{c}_{i}=\left[\begin{array}{l}
\mathbf{c}_{i}(1) \\
\mathbf{c}_{i}(2)
\end{array}\right], \quad \mathbf{c}_{i}(j) \text { is } L_{c} \times L, j=1,2,
$$

it is evident that

$$
\mathbf{g}_{i}=\left[\begin{array}{l}
\mathbf{g}(1) \\
\mathbf{g}(2)
\end{array}\right]=\mathbf{c}_{i} \mathbf{h}_{i}
$$

It is seen that $\left\{\mathbf{g}_{i}\right\}$ are uniquely determined by the unknown channel vectors $\left\{\mathbf{h}_{i}\right\}$.
If we desire the first user signal as a signal of interest, then, stacking data samples for two symbol period [1] provides

$$
\mathbf{X}(l)=\mathbf{g}_{1} s_{1}(n)+\mathbf{O}(n)
$$

where $\mathbf{O}(n)$ is given by

$$
\left[\begin{array}{cc}
\mathbf{g}_{1}(2) & \mathbf{0} \\
\mathbf{0} & \mathbf{g}_{1}(1)
\end{array}\right]\left[\begin{array}{c}
s_{1}(n-1) \\
s_{1}(n+1)
\end{array}\right]+\sum_{i=2}^{P} \mathbf{G}_{i} \mathbf{S}_{i}(n)+\mathbf{V}(n)
$$

Note that $\mathbf{O}(n)$ contains the ISI components for the signal of interest, the MUI, and the background noise. Furthermore, $\mathbf{G}_{i}$ and $\mathbf{S}_{i}$ are the interfering user signature waveform matrix and the input data matrix for the $i^{\text {th }}$, respectively [1].

In this paper, the structure of the desired user's signature waveform vector will be exploited to implement a self-recovering RAKE receiver. This structure enables the proposed receiver to combine all the multipath components and to minimize MUI.

\section{A SELF-RECOVERING RAKE RECEIVER}

The conventional RAKE receiver operates on the received signal with correlators known as "fingers". Finger outputs are coherently combined by using the knowledge of complex channel coefficients of each desired multipath component. This knowledge may be obtained from periodically transmitted training sequences. Linear Minimum Mean Square Error (LMMSE) receivers usually process all the users signals jointly. For the downlink receivers, only the desired user signal should be received while suppressing interference due to other users.

The minimum output energy (MOE) criterion was used in $[4,6]$ to derive blind adaptive receivers. It is well known that the MSE and MOE criterion lead to the same receivers and have the same performance under ideal conditions. Their performance depends on the adaptive implementations which may differ significantly.

\subsection{RAKE Receiver Based on CMA}

In this paper, we propose a self-recovering RAKE receiver, where the fingers are adapted jointly to suppress MAI. While the proposed RAKE receiver is similar to the decorrelating RAKE receiver in [5], the proposed receiver uses constant modulus property of the transmitted signal to speed up the converge of the weight vectors. The CMA is applied to the output signal which each finger of RAKE receiver is incoherently combined 
to produce. The proposed receiver has ability to do self-recovery in the sense that the receiver does not require the weighted sum of the finger outputs. After convergence the desired user signal can be obtained using the simple algorithm that was originally developed for single user antenna array applications [7].

In this paper, the receiver design problem using a constraint CMA algorithm is focused to determining weight vectors which provide estimates of the desired signal at each finger:

$$
z_{l}(n)=\mathbf{w}_{l}^{H} \mathbf{X}(n) .
$$

Vectors $\mathbf{w}_{l}$ are subject to a set of constrains

$$
\mathbf{w}_{l}^{H} \mathbf{C}=\mathbf{e}_{l}
$$

where $\mathbf{e}_{l}=[0, \cdots, 1, \cdots, 0]^{T}$ has 1 at $l$ th position. Fingers use the constrains optimization in order to suppress the interference.

Among the several CMA versions, we consider the Godard Algorithm [3] with cost function given as

$$
J\left(\mathbf{w}_{l}\right)=E\left[\left(\sum_{1}^{L}\left\|z_{l}\right\|^{2}-\delta\right)^{2}\right]
$$

where the constant $\delta$ sets the gain of the proposed RAKE receiver. This cost function is modified in the sense that the RAKE fingers are incoherently combined in order to produce the desired signal power. As shown in Figure 1, the cost function results in a CMA receiver which is a RAKE receiver where each constrained finger is adapted to suppress ISI and MAI. Search direction is restricted in the constrained subspace to find the projection of the gradient of the output energy onto the subspace orthogonal to $\mathbf{c}_{i}$. Using the orthogonal projection matrix given by

$$
\Pi_{\mathbf{c}_{i}}^{\perp}=\mathbf{I}-\mathbf{c}_{i}^{H}\left(\mathbf{c}_{i} \mathbf{c}_{i}^{H}\right) \mathbf{c}_{i}
$$

the weight vector for each finger is then adapted in accordance with the CMA [3]

$$
\mathbf{w}_{l}(n+1)=\mathbf{w}_{l}(n)-\mu \Pi_{\mathbf{c}_{i}}^{\perp} \mathbf{X}(n) \mathbf{z}^{H}(n) \mathbf{e}_{l}(n)
$$

where $\mu$ is the step-size parameter. $\mathbf{e}_{l}(n)$ is the error signal for the $l$ th finger defined by

$$
\mathbf{e}_{l}(n)=\sum_{1}^{L}\left|z_{l}(n)\right|^{2}-\delta
$$

Then, weight vectors $\left\{\mathbf{w}_{l}\right\}$ can be optimized by minimizing the constrained CMA cost function in equation (12).

After convergence, the finger outputs can be coherently combined using the simple algorithm developed in [7] for single user smart antenna systems. Using the array response vector analogy, the finger outputs can be rewritten as

$$
\mathbf{z}(n)=\left[\begin{array}{c}
z_{1}(n) \\
\vdots \\
z_{L}(n)
\end{array}\right]=\left[\begin{array}{c}
a_{1} \\
\vdots \\
a_{L}
\end{array}\right] \mathbf{s}_{1}(n)+\mathbf{n}(n) .
$$

We consider a simple combining with the estimate of finger response vector $\hat{\mathbf{a}}$. For $\mathrm{N}$ samples, we first estimate the power of each finger output $A_{l}=1 / N \sum_{n=1}^{N}\left|z_{l}(n)\right|^{2}$ for $l=1, \ldots, L$. Secondly, we construct an estimator by multiplying the finger outputs by the complex conjugated of the $k$ th finger whose output power is the maximum.

$$
\hat{\mathbf{a}}=\frac{1}{N_{t}} \mathbf{z}(n) z_{k}^{*}(n) .
$$

Then, the desired user signal can be recovered by $\left.s_{1} \hat{(} n\right)=$ $\hat{\mathbf{a} z}(n)$.

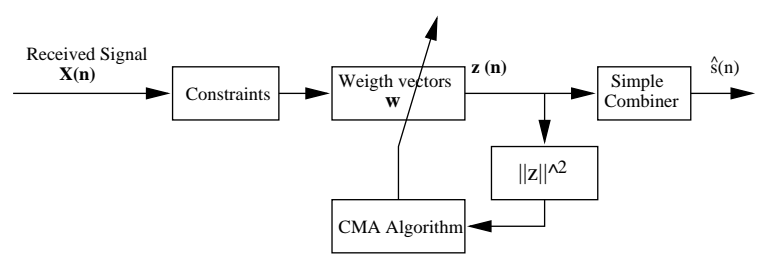

Figure 1: The proposed self-recovering RAKE receiver based on CMA

\section{SIMULATIONS}

To demonstrate the convergence behavior and the symbol error performance of the proposed constant modulus RAKE (CM-RAKE) receiver, we consider several examples of asynchronous CDMA systems.

We first consider a 8-user system with the spreading gain of 16. Signals undergo three-ray multipath fading with $\mathrm{SNR}=20 \mathrm{~dB}$. Furthermore, it is assumed that propagation delay of the desired signal is known by the receiver. Figure 2 compares the convergence behaviour of the adaptive D-RAKE [5], MOE [4] and CM-RAKE. Although we assumed that MOE receiver had the perfect knowledge of the effective signature waveform, the proposed RAKE receiver has better converge rate. In order to examine the fast convergence of the proposed method further, we consider average of MSEs between 1000 and 1100 iterations when the different number users are present in the system. Figure 3 shows the simulation results of averaged MSE with varying number of users. Last example in Figure 4 is given to show that 


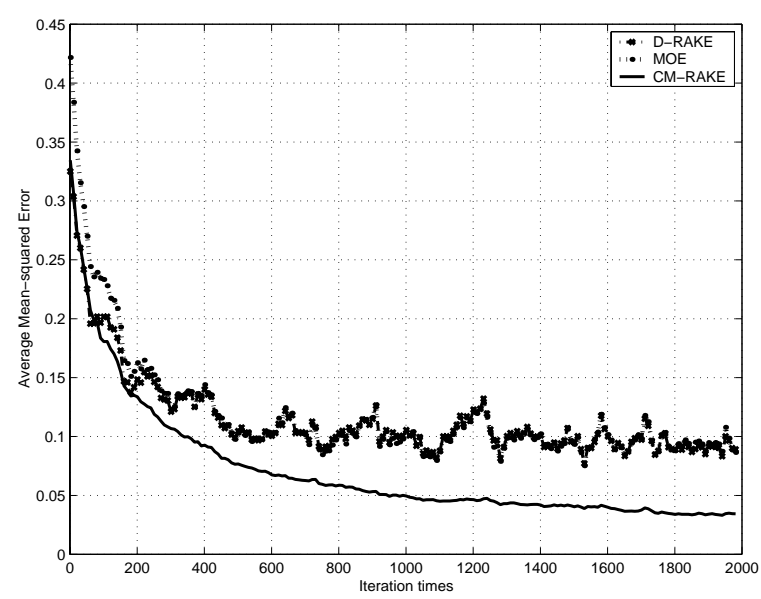

Figure 2: Convergence behaviour of the proposed CMRAKE as compared to adaptive D-RAKE and MOE receiver.

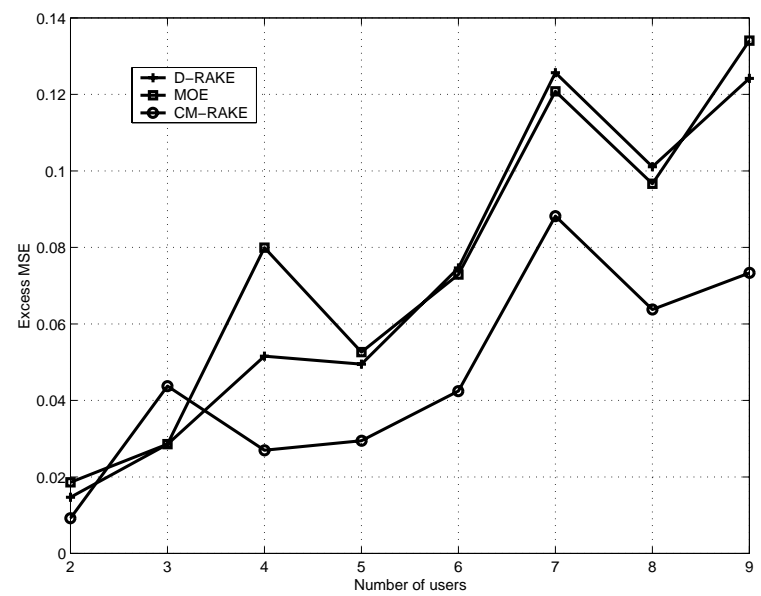

Figure 3: Averaged MSEs for the different adaptive receivers with varying number of users.

simple coherent combining algorithm can provide an open eye constellation as compared to adaptive RAKE receiver with the principal component combining after convergence.

\section{CONCLUSIONS}

In this paper, we have proposed and demonstrated a self-recovering RAKE receiver based on the constant modulus algorithm (CMA). The receiver has better convergence rate that other blind adaptive receivers. The proposed receiver can directly operate on the combined output powers, thus, it eliminates the need for an immediate second stage to obtain the demodulated signal. The signal can be demodulated after convergence
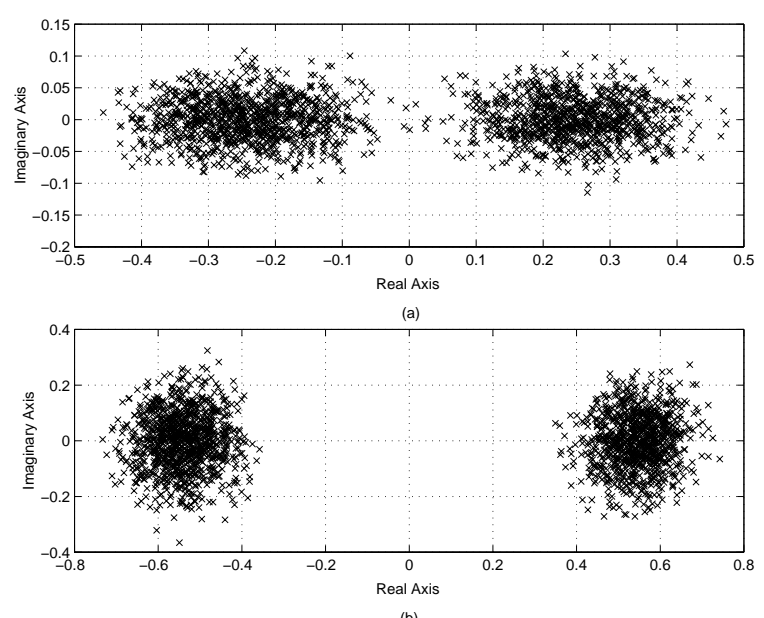

Figure 4: Signal constellations of (a) adaptive D-RAKE and (b) the proposed CM-RAKE after convergence in 8-user system.

using the simple coherent combining algorithm.

\section{REFERENCES}

[1] M. Torlak and G. Xu, "Blind multi-user channel estimation in asynchronous CDMA systems", IEEE Trans. on Signal Processing, vol. 45, no. 1, pp. 137147, Jan. 1997.

[2] H. Liu and G. Xu, "A subspace method for signature waveform estimation in synchronous CDMA systems", IEEE Trans. on Communications, vol. 44, no. 10, pp. 1346-1354, Oct. 1996.

[3] S. Haykin, Adaptive Filter Theory, Prentice-Hall, Englewood Cliffs, NJ, second edition, 1991.

[4] M. L. Honig, U. Madhow, and S. Verdú, "Blind adaptive multiuser detection", IEEE Trans. on Information Theory, vol. 41, no. 4, pp. 944-962, July 1995.

[5] H. Liu and K. Li, "A decorrelating RAKE receiver for CDMA communications over frequencyselective fading channels", IEEE Trans. Communications, vol. 47, no. 7, pp. 1036-1045, July 1999.

[6] M. K. Tsatsanis, "Inverse filtering criteria for CDMA systems", IEEE Trans. on Signal Processing, vol. 45, no. 1, pp. 102-112, Jan. 1997.

[7] S. Kim and G. Xu, "Antenna gain for adaptive smart antenna systems", in Proc. IEEE Vehicular Technology Conf., Phoenix, AZ, May 1997, vol. 2, pp. 1168-1172. 